\title{
From high to low malaria transmission in Zanzibar-challenges and opportunities to achieve elimination
}

\author{
A. Björkman ${ }^{1 *}$, D. Shakely ${ }^{1,2+}$, A. S. Ali ${ }^{3}$, U. Morris ${ }^{1}$, H. Mkali ${ }^{4}$, A. K. Abbas ${ }^{3 \wedge}$, A-W Al-Mafazy ${ }^{3}$, K. A. Haji ${ }^{3}$, J. Mcha ${ }^{3}$, \\ R. Omar ${ }^{3}$, J. Cook ${ }^{1,5}$, K. Elfving ${ }^{1,6}$, M. Petzold ${ }^{7}$, M. C. Sachs ${ }^{8}$, B. Aydin-Schmidt ${ }^{1}$, C. Drakeley ${ }^{5}$, M. Msellem ${ }^{9}$ and \\ A. Mårtensson ${ }^{10}$
}

\begin{abstract}
Background: Substantial global progress in the control of malaria in recent years has led to increased commitment to its potential elimination. Whether this is possible in high transmission areas of sub-Saharan Africa remains unclear. Zanzibar represents a unique case study of such attempt, where modern tools and strategies for malaria treatment and vector control have been deployed since 2003.

Methods: We have studied temporal trends of comprehensive malariometric indices in two districts with over 100,000 inhabitants each. The analyses included triangulation of data from annual community-based cross-sectional surveys, health management information systems, vital registry and entomological sentinel surveys.

Results: The interventions, with sustained high-community uptake, were temporally associated with a major malaria decline, most pronounced between 2004 and 2007 and followed by a sustained state of low transmission. In 2015, the Plasmodium falciparum community prevalence of $0.43 \%$ (95\% Cl $0.23-0.73$ ) by microscopy or rapid diagnostic test represented $96 \%$ reduction compared with that in 2003. The P. falciparum and P. malariae prevalence by PCR was $1.8 \%$ ( $95 \% \mathrm{Cl} 1.3-2.3$ ), and the annual P. falciparum incidence was estimated to 8 infections including 2.8 clinical episodes per 1000 inhabitants. The total parasite load decreased over 1000-fold (99.9\%) between 2003 and 2015. The incidence of symptomatic malaria at health facilities decreased by $94 \%$ with a trend towards relatively higher incidence in age groups $>5$ years, a more pronounced seasonality and with reported travel history to/from Tanzania mainland as a higher risk factor. All-cause mortality among children < 5 years decreased by $72 \%$ between 2002 and 2007 mainly following the introduction of artemisinin-based combination therapies whereas the main reduction in malaria incidence followed upon the vector control interventions from 2006. Human biting rates decreased by 98\% with a major shift towards outdoor biting by Anopheles arabiensis.
\end{abstract}

Conclusions: Zanzibar provides new evidence of the feasibility of reaching uniquely significant and sustainable malaria reduction (pre-elimination) in a previously high endemic region in sub-Saharan Africa. The data highlight constraints of optimistic prognostic modelling studies. New challenges, mainly with outdoor transmission, a large asymptomatic parasite reservoir and imported infections, require novel tools and reoriented strategies to prevent a rebound effect and achieve elimination.

\footnotetext{
* Correspondence: anders.bjorkman@ki.se

${ }^{+}$A. Björkman and D. Shakely contributed equally to this work.

Deceased

${ }^{1}$ Department of Microbiology, Tumor and Cell Biology, Karolinska Institutet,

Solnavägen 9, SE-171 77 Stockholm, Sweden

Full list of author information is available at the end of the article
}

(c) The Author(s). 2019 Open Access This article is distributed under the terms of the Creative Commons Attribution 4.0 International License (http://creativecommons.org/licenses/by/4.0/), which permits unrestricted use, distribution, and reproduction in any medium, provided you give appropriate credit to the original author(s) and the source, provide a link to the Creative Commons license, and indicate if changes were made. The Creative Commons Public Domain Dedication waiver (http://creativecommons.org/publicdomain/zero/1.0/) applies to the data made available in this article, unless otherwise stated. 


\section{Background}

Substantial progress has been made globally in reducing the malaria burden during last decade [1] following the implementation and scaling up of vector control and effective treatment. This has led to increased international commitment to malaria elimination in several endemic areas [2, 3]. Elimination is considered feasible - and indeed has been achievedusing existing tools in areas of low to moderate transmission [4]. Whether elimination can be achieved in previously moderate to high transmission areas, however, remains unclear. According to the World Health Organization (WHO), "demonstrated technical feasibility in such eco-epidemiological settings is required [5]." Zanzibar with historically high malaria transmission $[6,7]$ may represent a rather unique, useful and valuable case study of such endeavour.

Zanzibar was among the first in sub-Saharan Africa to roll out wide-scale modern control interventions [8], i.e. the use of artemisinin-based combination therapy (ACT) in $2003 / 2004$ followed by long-lasting insecticidal nets (LLINs), indoor residual spraying (IRS) and rapid diagnostic tests (RDTs) in 2005/2006 (Table 1).

We herein report to what extent reduction in malaria transmission towards elimination has been achieved a decade after the intensified malaria control and why and where obstacles have emerged. Intervention uptake and temporal trends of a comprehensive variety of malariometric indices are presented including molecular and serological surveillance methods.

\section{Methods}

\section{Study sites and malaria control interventions}

Zanzibar (population 1.3 million) is an archipelago with two main islands about $30 \mathrm{~km}$ from mainland Tanzania (Fig. 1).
The study was conducted in two rural districts, North A (Unguja island) and Micheweni (Pemba island), each with a population of approximately 100,000 people. The two districts, selected in 2003, were considered to be representative (e.g. mainly rural) for each of the two main islands of Zanzibar. Each of the two districts included one public district hospital, 13 public health care facilities and two private clinics. There is access to a public health care facility within $5 \mathrm{~km}$ distance throughout Zanzibar.

Malaria transmission has historically been holoendemic with a trend towards hyperendemicity in the 1990s $[6,7,9]$ and perennial with two peaks associated with seasonal rainfalls in March-June and October-November. When the new interventions started in 2003, Plasmodium falciparum was the pre-dominant malaria species and Anopheles gambiae sensu lato, An. funestus and $A n$. coustani were the main vectors [7].

The introduction of new malaria control interventions and strategies are presented in Table 1. The interventions were implemented by the Zanzibar Malaria Control Programme (ZMCP), which became the Zanzibar Malaria Elimination Programme (ZAMEP) in August 2013.

\section{Cross-sectional surveys}

Nine cross-sectional household surveys were conducted in May-June between 2003 and 2015. The 2003 exploratory survey was conducted using a two-stage cluster sample technique. First, shehias (smallest administrative unit) and then the households were randomly selected from the sampling frame obtained from the Office of Government Statistics, Zanzibar [8]. This first survey included 625 households. Sample size calculations for the

Table 1 Implementation of malaria control tools/strategies in Zanzibar between 2002 and 2016

\begin{tabular}{|c|c|}
\hline Year, month & Interventions \\
\hline 2002, November & New antimalarial treatment policy: ACT; 1st line: $A S A Q, 2$ nd line: $A L$ \\
\hline 2003, September & ACT deployment in all public health facilities. \\
\hline 2004 & ITN distribution, geographically focused Intermittent preventive treatment in pregnancy (IPTp) \\
\hline 2005, September & LLIN universal distribution to all children $<5$ years and pregnant women \\
\hline 2006, July & IRS (pyrethroid) aiming at annual universal coverage, in March before the main transmission season (after 2006) \\
\hline 2006 & $\begin{array}{l}\text { RDT provision to all public health facilities. LLIN provision initiated to all pregnant women and infants ( } 9 \text { months old) in } \\
\text { MCH clinics }\end{array}$ \\
\hline 2008 & LLIN universal distribution-two nets per household \\
\hline 2009 & $\begin{array}{l}\text { New antimalarial treatment policy: 1st line: ASAQ, 2nd line: quinine Weekly reporting of malaria cases by mobile phone } \\
\text { from health care facilities (MEEDS) }\end{array}$ \\
\hline 2012 & $\begin{array}{l}\text { LLIN universal distribution-two nets per household IRS policy change: targeting hotspots only (carbamate 2012-2014, } \\
\text { pirimiphos-methyl 2015-) Malaria case investigation and reactive household RDT screening and LLIN distribution }\end{array}$ \\
\hline 2015 & $\begin{array}{l}\text { RDT and ACT provision to private health facilities (AMFm programme) Intermittent screening and treatment in pregnancy } \\
\text { (ISTp) replacing IPTp }\end{array}$ \\
\hline 2016 & Larviciding in few selected sites New antimalarial treatment policy: ACT + primaquine (single low dose) \\
\hline
\end{tabular}

ACT artemisinin-based combination therapy, ASAQ artesunate-amodiaquine, AL arthemeter-lumefantrine, ITN insecticide-treated net, IRS indoor residual spraying, RDT rapid diagnostic test, LLIN long-lasting insecticidal net, MEEDS malaria early epidemic detection system, AMFm affordable medicines for malaria, MCH mother and child health 


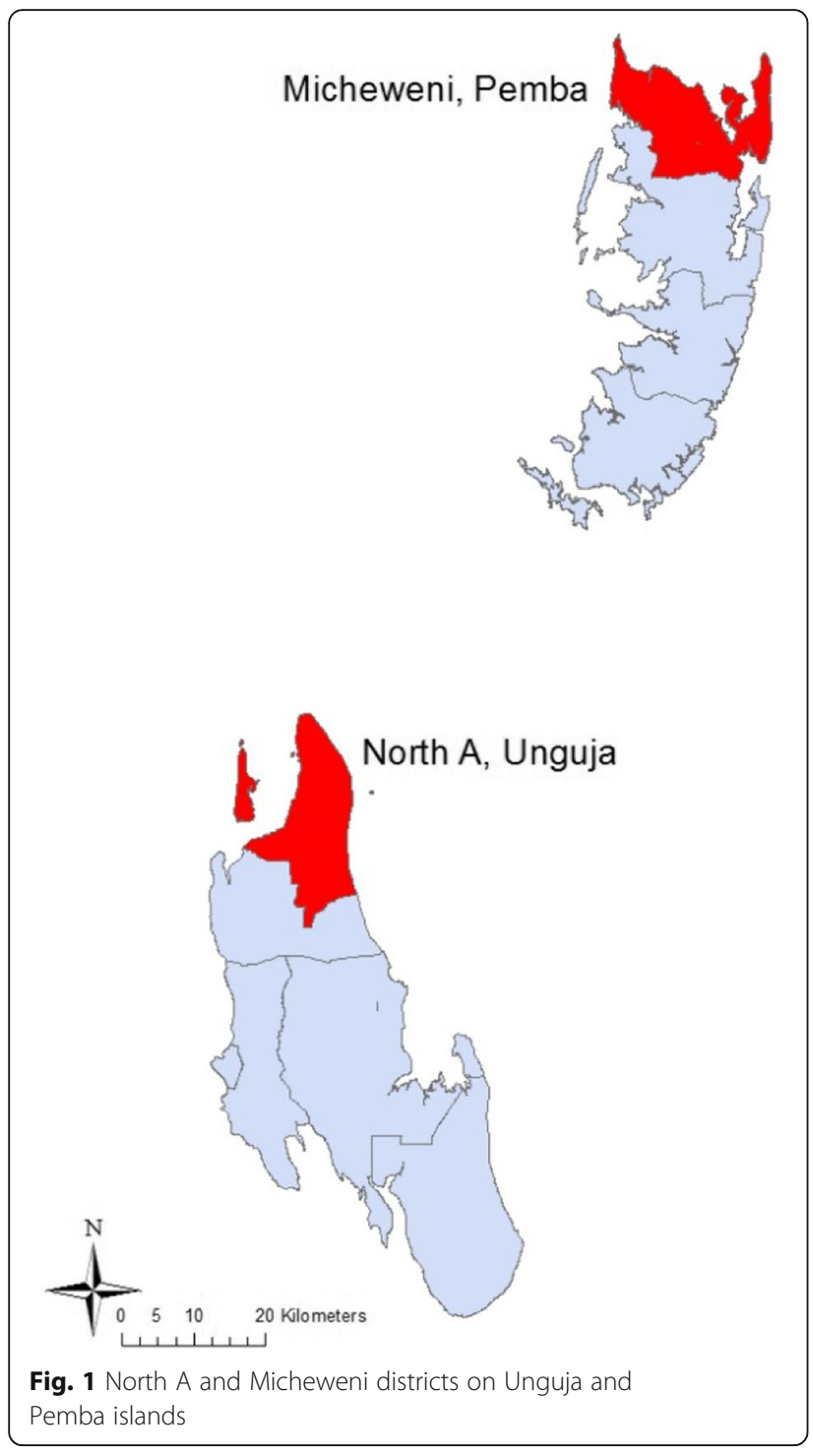

follow-up surveys were adjusted according to changing malaria prevalences. Those conducted in 2005 to 2007 were based on the proportion of children under five with malaria parasitaemia in $2003,9 \%$, and an assumed relative error of $20 \%$. The calculated number of households to be included was 490 after adjusting for a design effect of 2. The same shehias were selected in the follow-up surveys, but each time with randomly selected households in proportion to the shehia size. From 2009 onwards, assuming $1 \%$ of households with at least one member being parasite positive and $95 \% \mathrm{CI}$ of $+/-1 \%$ and $20 \%$ household members absent or refusing participation, the targeted sample sizes were 350 households.

Trained health personnel visited the selected households and conducted interviews (history of recent travel, access to health care, use of LLIN, IRS coverage, etc.) and blood sample collections (for malaria diagnosis by blood smear or RDT, PCR and serological analyses, etc.) upon written informed consent or proxy consent from legal guardians for children $\leq 15$ years.

\section{Laboratory methods}

Microscopic examination of Giemsa-stained thick blood smears was used for parasitological screening in the cross-sectional surveys 2003-2009. A P. falciparum-specific histidine-rich-protein 2 (HRP2)-based RDT (Paracheck) was used in 2011 followed by a combo RDT detecting both HRP2 and pan-Plasmodium lactate dehydrogenase in 2013 (SD-Bioline) and 2015 (Malaria ag combo).

Plasmodium detection by PCR was conducted on dried filter paper blood spots after DNA extraction by Chelex ${ }^{\circ}$ boiling method [10]. Cytb SYBR Green qPCR followed by restriction fragment length polymorphism analysis [11] was used for parasite screening and species determination. Parasite density was estimated by qPCR [12].

Antibody responses to three P. falciparum blood stage proteins (AMA-1, MSP-1 and GLURP) were determined by ELISA on blood spots from the cross-sectional surveys in 2009 and 2015 Mixture models were fitted to OD ELISA responses assuming two Gaussian distributions and the seroprevalence cut-off defined as the mean plus 3 standard deviations of the narrow, negative distribution [13]. Models were fitted separately for each antigen and combined to generate an overall seroprevalence if positive to one or more antigen. Seroconversion rates (SCRs) were estimated using reverse catalytic conversion models.

\section{Health facility and vital registry}

Health facility data were collected monthly from the 26 public health care centres in the two districts (13 each) throughout the study period (1999-2015) using the Health Management Information System (HMIS) of the Ministry of Health. This included numbers of malariasuspected patients, if malaria-tested and if confirmed by microscopy or RDT as well as individual characteristics of patients (age, sex, locality, etc.). In 2009, the HMIS data reporting was reinforced by the "malaria early epidemic detection system" (MEEDS) with weekly reports through a mobile phone reporting system (Table 1). Finally, from 2011, the "malaria case notification system" (MCN) was implemented, including added epidemiologyrelated information such as malaria risk factors (use of LLINs, IRS coverage, recent travel history, etc.) [14]. Parasitological confirmation by microscopy or RDT was not compulsory for all febrile patients until 2007. We therefore used positivity rates of those tested, rather than absolute malaria cases in our trend analyses.

Vital registry records on total births and deaths (19982014) were obtained from District Commissioner's Office. 
Demographic data were obtained from the Tanzanian National Population and Housing Census.

\section{Entomological and meteorological data}

Entomological data were collected from 22 sentinel sites (shehias) on Unguja and Pemba islands from 2005 onwards including North A and Micheweni districts. This included collections of adult mosquitoes indoors (18-06 h) and outdoors (18-24h) in randomly selected households and larvae in selected breeding grounds. Indoor and outdoor human biting rates (HBRs) were determined by the human-landing catch method. In each sentinel site, two human volunteer baits would sit in- and outside two houses for four consecutive nights each month. Additional collections by pyrethrum spray light-traps and pit traps were also performed in some sites. Mosquitoes were stored in paper cups for species identification by PCR [15]. Sporozoites in the salivary glands were identified by ELISA [16] Insecticide resistance testing was conducted using the WHO guidelines [17]. Anopheles larva were collected, allowed to develop to adults, then exposed to the insecticides $[15,18]$.

Records of monthly rainfall for North A (1999-2015) and Micheweni (2005-2015) were obtained from Zanzibar Ministry of Communication and Transport through the Tanzania Meteorological Agency.

\section{Data management and analyses}

Survey data were entered and validated using Microsoft Access and Excel. Recent surveys were conducted via tablets using Open Data kit. Statistical analyses were performed using STATA versions 12 and 13, and R version 3.2.2 ( $\mathrm{R}$ Foundation for Statistical Computing, Vienna, Austria). Some data represent aggregate data from published articles $[8,15,18,19]$ and/or annual malaria reports of ZMCP/ZAMEP [14].

Pearson correlation coefficients were calculated to assess linear relationships between monthly rainfall and malaria incidence. Poisson regression model was used to assess malaria incidence and interaction of age $(<5$ and $>5$ years of age) and calendar year. Exact binomial tests were used where numbers were small $(<5)$. Otherwise, the normal approximation was used to test for differences in proportions. Trends in prevalences over time were tested using binomial logistic regression on the aggregate counts. Fisher's test was used to assess differential reduction between malaria species. Logistic regression was used to assess the associations between risk factors and infection.

Serological conversion rate (SCR) was used to estimate the force of infection (incident malaria cases per population time) using maximum likelihood methods [20]. Temporal change in SCR was identified using profile likelihood plots and likelihood ratio tests against models with no change $[3,21]$.

Annual parasite incidence or index (API), defined as annual malaria cases per year per 1000 inhabitants, was based on any parasite-positive infection, whether symptomatic or not, whereas "clinical API" was based on reported malaria confirmed clinical patients only.

Reproductive control rate/ratio (Rc) is normally defined as number of new infections occurring from each index infection. We have estimated this concept as "annual Rc" in year X by calculating "annual incidence ratio" (incidence year X/incidence year $\mathrm{X}-1$ ) or "annual prevalence ratio" (prevalence year $\mathrm{X} /$ prevalence year $\mathrm{X}$ $-1)$.

The sporozoite rate was based on human light-trap catches throughout the year. However, after the intensified vector control in 2006, the sporozoite rates became too low for a precise estimate. The rate was then extrapolated from the mean human asexual parasite prevalence and density, assuming a linear correlation of the parasite prevalence and non-linear correlation of the parasite density [22] with the sporozoite rate (and infectiousness). The annual entomological inoculation rate (EIR) was determined as the mean HBR $\times$ mean sporozoite rate $\times 365$ days.

\section{Results}

\section{Intervention uptake}

ACTs and RDTs were available in all health facilities without any documented stock-out periods since their respective implementation. There was high reported use of insecticide-treated net (ITN)/LLIN in all age groups in the cross-sectional surveys between 2006 and 2015 in Micheweni and North A (mean 68\% and 74\%, respectively) although children $<5$ years were more likely to use ITN/LLIN (81\%) than individuals $\geq 5$ years $(69 \%)(p<$ 0.0001) (Additional file 1: Figure S1). After the first IRS in July 2007, annual IRS was implemented in March each year prior to the expected main rains and transmission season. The mean proportion of households reporting having had IRS within the previous year was $87.6 \%$ from 2008 to 2011 in the two districts, 91\% in Micheweni and $85 \%$ in North $\mathrm{A}$-with annual ranges from 80 to 92\% (Additional file 1: Figure S1). In 2013 and 2015 when IRS became more targeted, mainly to hotspot areas, the mean coverages were $79 \%$ in Micheweni and $54 \%$ in North A.

\section{Community-based parasite surveys}

The overall microscopy- or RDT-determined parasite prevalences of all ages at the cross-sectional surveys are shown in Table 2 and Additional file 2: Table S1. Only $P$. falciparum infections were detected. In 2015, the prevalences were $0.6 \%$ in Micheweni and $0.3 \%$ in North A 
Table 2 Community prevalences of asexual P. falciparum parasitaemia by microscopy or RDT; all age groups in May/June

\begin{tabular}{|c|c|c|c|c|c|c|}
\hline \multirow[t]{2}{*}{ Year } & \multicolumn{3}{|c|}{ Micheweni district } & \multicolumn{3}{|c|}{ North A district } \\
\hline & Tested & Positive & Positivity rates $(95 \% \mathrm{Cl})$ & Tested & Positive & Positivity rates $(95 \% \mathrm{Cl}$ \\
\hline 2003 & 1189 & 172 & $14.5 \%(12.5-16.6)$ & 2167 & 174 & $8.0 \%(6.9-9.3)$ \\
\hline 2005 & 1241 & 135 & $10.9 \%(9.2-2.7)$ & 1503 & 48 & $3.2 \%(2.4-4.2)$ \\
\hline 2006 & 1182 & 56 & $4.7 \%(3.6-6.1)$ & 1433 & 12 & $0.8 \%(0.4-1.5)$ \\
\hline 2007 & 1575 & 15 & $1.0 \%(0.5-1.6)$ & 1499 & 0 & $0.0 \%(0.0-0.2)$ \\
\hline 2008 & 2091 & 10 & $0.5 \%(0.2-0.9)$ & 1746 & 4 & $0.2 \%(0.1-0.6)$ \\
\hline 2009 & 1539 & 0 & $0.0 \%(0-0.2)$ & 1163 & 0 & $0.0 \%(0.0-0.3)$ \\
\hline $2011^{*}$ & 1271 & 10 & $0.8 \%(0.4-1.4)$ & 1561 & 2 & $0.1 \%(0.0-0.5)$ \\
\hline $2013^{*}$ & 1579 & 7 & $0.4 \%(0.2-0.9)$ & 1447 & 3 & $0.2 \%(0.0-0.6)$ \\
\hline $2015^{*}$ & 1515 & 9 & $0.6 \%(0.3-1.1)$ & 1497 & 4 & $0.3 \%(0.1-0.7)$ \\
\hline
\end{tabular}

*Malaria diagnosis by RDT instead of blood slide microscopy

(mean 0.43\%, 95\% CI 0.23-0.73). This represents 95.9\% and $96.6 \%$ (mean 95.8\%; 24-fold) reductions in parasite prevalences, respectively, as compared with 2003. The main reduction occurred during 2006 and 2007 following the introduction of LLINs and IRS, after which a low prevalence was maintained, averaging $0.45 \%$ in Micheweni and $0.18 \%$ in North A. P. falciparum gametocyte carriage was detected at low prevalences $(<1 \%)$ in 20032006, but not in 2008-2009. The P. falciparum parasite densities in detected infections also declined significantly (Table 4). In the two districts combined, the geometrical mean parasitaemias were in 2003/51,147 parasites/ $\mu \mathrm{l}$ (range 80 to 341,400 ) and in 2013/15,230 parasites/ $\mu \mathrm{l}$ (range 53 to 770 ) among microscopy- and RDT (> 50 parasites $/ \mu \mathrm{l})$-detectable infections.

The community parasite prevalences determined by PCR are presented in Table 3. Only P. falciparum and $P$. malariae were detected in the five surveys 2005-2015. The overall ratios between microscopy/RDT- and PCR-detectable malaria infections were 1:3 in 2005 and 1:8 in 2009-2015 combined. In 2015, the overall mean parasite prevalence in the two districts was $1.8 \%$ (95\% CI 1.3-2.3\%). This represents an estimated reduction of 92.7\% (14-fold) compared with 2003. In the four surveys 2009-2015, the mean PCR determined parasite prevalence in Micheweni (3.2\%) was 2.1 times higher than in North A
$(1.5 \%)(p<0.001)$. However, there were opposite trends $(2009-2015)$ in the prevalences, decreasing in Micheweni while increasing in North A $(p<0.001)$. There was no significant shift in relative prevalence from younger children to older age groups over time and throughout the study, the highest prevalences were seen in children 5-14 years. In 2005 , the prevalences were $13.1 \%$ in children $<5$ years, $36.4 \%$ in $5-14$ years and $15.9 \%$ in $>14$ years. In 2015 , the prevalences were $1.9 \%, 2.0 \%$ and $1.7 \%$, respectively.

In the two districts combined, the main reduction of PCR-detected P. falciparum occurred from 2005 (20.9\%) to $2009 / 11$ (2.7\%) followed by $2.0 \%$ in $2013-2015$ whereas the main reduction of $P$. malariae occurred later, i.e. from $2005(2.3 \%)$ and $2009-2011$ (1.1\%) to 2013-2015 (0.2\%). Hence, the proportions of $P$. malariae infections among detected infections were $11 / 100(11 \%)$ in $2005,61 / 248$ $(25 \%)$ in $2009-2011$ and $14 / 122(11 \%)$ in $2013-2015$ ( $p=$ 0.0004 for 2009-2011 vs. 2013-2015). The geometric mean parasite densities in 2009-2015 as determined by qPCR were 27 (range $<1-7825$ ) parasites/ $\mu$ lamong $P$. falciparum mono infections and 4 (range $<1-29$ ) parasites/ $\mu \mathrm{l}$ among $P$. malariae mono infections.

\section{Health facility data}

Monthly parasitologically confirmed malaria diagnoses from health facility outpatients reported between 1999

Table 3 Community parasite prevalences (P. falciparum and P. malariae) by PCR; all age groups in May/June

\begin{tabular}{|c|c|c|c|c|c|c|}
\hline \multirow[t]{2}{*}{ Year } & \multicolumn{3}{|c|}{ Micheweni district } & \multicolumn{3}{|l|}{ North A district } \\
\hline & Samples tested & Positive & Positivity rate $(95 \% \mathrm{Cl})$ & Samples tested & Positive & Positivity rate $(95 \% \mathrm{Cl})$ \\
\hline 2005 & 190 & 53 & $27.9 \%(21.7-34.9)$ & 288 & 47 & $16.3 \%(12.2-21.1)$ \\
\hline 2009 & 1410 & 72 & $5.1 \%(4.0-6.4)$ & 1013 & 9 & $0.9 \%(0.4-1.7)$ \\
\hline 2011 & 1378 & 45 & $3.3 \%(2.4-4.4)$ & 1599 & 22 & $1.4 \%(0.9-2.1)$ \\
\hline 2013 & 1575 & 42 & $2.7 \%(1.9-3.6)$ & 1448 & 26 & $1.8 \%(1.2-2.6)$ \\
\hline 2015 & 1519 & 26 & $1.7 \%(1.1-2.4)$ & 1497 & 28 & $1.9 \%(1.2-2.6)$ \\
\hline
\end{tabular}

Linear trends in positivity rates from 2009 to 2015 were statistically significant in Micheweni district (decreasing $p<0.001$ ) and in North A (increasing $p=0.036$ ). These trends differed by district (interaction) $(p<0.001)$ 
and 2015 are presented in Fig. 2a-c and Additional file 2: Table S2. The overall reduction in microscopy/RDT positivity rate between 2002 and 2015 was 95.7\% (from 46.2 to $2.0 \%$ ) in Micheweni and $89.7 \%$ (from 23.3 to $2.4 \%$ ) in North A. This corresponds to 23- and 10-fold reductions in the respective districts and a mean reduction of $94.2 \%$ (Table 4).
The main reduction occurred from 2005 when vector control was implemented in addition to ACT which is also consistent with the observed cross-sectional parasite prevalences. Between 2005 and 2008, the prevalence reduction was approximately 10 -fold, i.e. with an annual reproductive control rate (Rc) of approximately 0.50, when estimated as "annual prevalence ratio". The annual
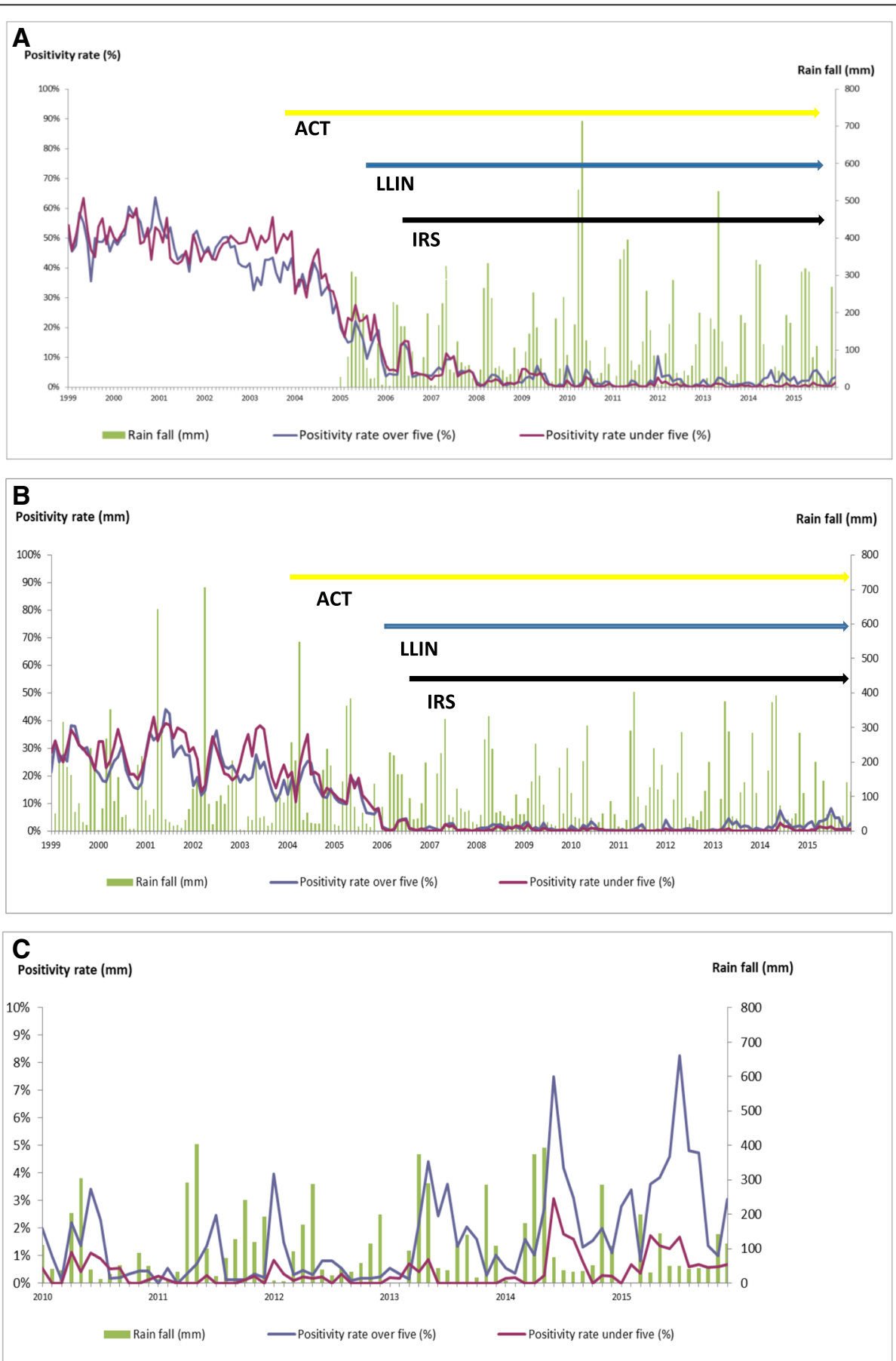

Fig. 2 Malaria positivity rates among $<5$ and $\geq 5$ febrile patients in relation to monthly rainfall and interventions. a Febrile patients attending health care facilities in Micheweni district. b, c Febrile patients attending health care facilities in North A district 
Table 4 Malaria-related indices before interventions (2002-2003) compared to 2015; both districts combined

\begin{tabular}{|c|c|c|c|}
\hline & $2002 / 3$ & 2015 & Reduction \% (X-fold) \\
\hline \multicolumn{4}{|l|}{ Fever patients in health care facilities } \\
\hline Parasite prevalence by microscopy/RDT & $38.2 \%(95 \%$ Cl 37.5-39.0) & $2.2 \%,(95 \% \mathrm{Cl} 2.0-2.3)$ & $94.2 \%(17)$ \\
\hline \multicolumn{4}{|l|}{ Community-based cross-sectional surveys } \\
\hline Parasite prevalence by microscopy/RDT & $10.3 \%(95 \%$ Cl 9.3-11.4) & $0.43 \%(95 \% 0.23-0.73)$ & $95.8 \%(24)$ \\
\hline Parasite prevalence by PCR & $24.8 \%(95 \%$ Cl 23.4-26.3) & $1.8 \%(95 \% \mathrm{Cl} 1.3-2.3)$ & $92.7 \%(14)$ \\
\hline \multicolumn{4}{|l|}{ Parasite densities } \\
\hline Among microscopy positive or $\geq 50 \mathrm{par} / \mu \mathrm{l}$-geometrical mean $\mathrm{par} / \mu \mathrm{l}$ & 1135 (Range 115-149,000) & 161(Range 53-770) & $85.8 \%(7)$ \\
\hline Among all—arithmetical mean par/ $\mu l$ & $450^{*}$ & 0.34 & $99.9 \%(1324)$ \\
\hline Seroconversion rate per year & $11 \%(95 \% \mathrm{Cl} 8-13)$ & $0.8 \%(95 \%$ Cl 0.6-1.1) & $92.1 \%(14)$ \\
\hline Crude under 5 child mortality per year** & $1.01 \%(95 \%$ Cl 0.84-1.19) & $0.36 \%(95 \%$ Cl $0.28-0.48)$ & $64.4 \%(2.8)$ \\
\hline Human biting rate per person night*** & $12.44^{*}$ & 0.27 & $97.8 \%(46)$ \\
\hline Entomological inoculation rate (infective bites/year) ${ }^{* * *}$ & $136^{*}$ & 0.05 & $>99.9 \%(2720)$ \\
\hline
\end{tabular}

*In 2005 before intensified vector control

**North A only

***Mean of indoors and outdoors

number of parasitologically confirmed malaria patients in the two districts went from 3528 in 2005, approximately sixfold down to 578 in 2008, with an annual Rc of approximately 0.55 . From 2003 to 2008, the average annual Rcs were estimated to 0.50 and 0.63 . From 2008 onwards, relatively low microscopy/RDT positivity rates were observed with averages of $1.8 \%$ in Micheweni and $1.2 \%$ in North A. The estimated average annual incidences ("Clinical APIs") during this period were 3.6 and 2.0 (mean 2.8) per 1000 inhabitants for Micheweni and North A, respectively. Interestingly, there were opposite trends in the two districts from $2012(p>0.001)$ with continued decreasing parasite rates in Micheweni $(p<$ $0.01)$ and increasing rates in North A $(p<0.01)$ (Additional file 2: Table S2).

A relative shift in parasite positivity rates was observed towards older age groups between 2002 and 2015 (Fig. 2a-c). Hence, the malaria patients $<5$ years of age represented $47.2 \%(3067 / 6502)$ of all malaria patients in 2002 and $17.3 \%(125 / 721)$ in $2015(p<0.001)$ in the two districts. There was also a shift from perennial to seasonal malaria (during the main rains) (Fig. 2a-c). Hence, 2001 and 2002, 20\% (374/1891) and 28\% (380/1377) of reported malaria cases occurred during the 2 months with highest numbers whereas in 2014 and 2015 the corresponding figures were $44 \%(142 / 326)$ and $41 \%(128 /$ $316)$, respectively, i.e. a 1.8 -fold increase comparing $2001 / 2002$ to $2014 / 2015(p<0.001)$.

In Micheweni, prevalences of reported fever within 14 days prior to the cross-sectional surveys were $19 \%$ in 2003 and 7\% in $2009(p<0.01)$ whereas in North A reported fever remained consistent around $12 \%$. Both districts, reported high levels ( $>90 \%$ ) of public facility use, consistent with findings throughout Zanzibar from health management information systems in 2009 and 2010. The health facility reporting system lately included several private facilities and therefore most clinical malaria episodes in the study area.

\section{Risk factors for malaria infections}

Risk factors associated with RDT-positivity among fever patients attending public health facilities, representing clinical malaria infections, compared to healthy controls, were assessed in 2015 (Table 5). History of recent travel (<1 month) outside Zanzibar (mainly Tanzania mainland) was reported by 118 (44.2\%) malaria-positive females and 244 (51.3\%) malaria-positive males. Reported travel outside Zanzibar was associated with increased adjusted ORs of 85.6 (95\% CI 50.9-151.4) for females and 60.3 (95\% CI 38.9-96.5) for males, while travel within Zanzibar was not associated with any increased risk (adjusted OR, 95\% CI 0.7-1.6). The temporal trends of the malaria cases reporting travel were similar to those not reporting travel, i.e. highly seasonal. Not sleeping under a bed net was also associated with increased risk of RDT positivity, adjusted OR 4.4 (95\% CI 3.9-6.0), whereas IRS did not affect malaria risk.

Risk factors associated with asymptomatic PCR positivity were also assessed in the 2015 cross-sectional survey (Table 5). There was no evidence to suggest that malaria infection was significantly associated with travel outside or inside Zanzibar (past month or within last year) or with use of any vector control.

\section{All-cause child mortality}

The Vital Registry reported all cause annual mortality rate among children $<5$ years in North A district was $1.01 \%$ in $2001 / 2002$ before the malaria control 
Table 5 Risk factors in malaria infected asymptomatic and symptomatic individuals in both districts combined in 2015

\begin{tabular}{|c|c|c|c|c|c|c|}
\hline & $\begin{array}{l}\text { Healthy, malaria-negative } \\
\text { controls**** }\end{array}$ & $\begin{array}{l}\text { Asymptomatic PCR } \\
\text { positive }\end{array}$ & $\begin{array}{l}\text { Unadjusted OR } \\
(95 \% \mathrm{Cl})\end{array}$ & $\begin{array}{l}\text { Symptomatic RDT } \\
\text { positive }\end{array}$ & $\begin{array}{l}\text { Unadjusted OR } \\
(95 \% \mathrm{Cl})\end{array}$ & $\begin{array}{l}\text { Adjusted OR } \\
(95 \% \mathrm{Cl})\end{array}$ \\
\hline Total & $2938(100 \%)$ & $53(100 \%)$ & - & $743(100 \%)$ & - & 1 (Ref) \\
\hline Male & 1202 (41\%) & $29(55 \%)$ & 1 (Ref) & $476(64 \%)$ & 1 (Ref) & 1 (Ref) \\
\hline Female & 1736 (59\%) & $24(45 \%)$ & 0.6 (0.3 to 1.0$)$ & 267 (36\%) & 0.4 (0.3 to 0.5$)$ & 0.5 (0.4 to 0.6$)$ \\
\hline Slept under net-yes* & 2062 (70\%) & $33(62 \%)$ & 1 (Ref) & $286(38 \%)$ & 1 (Ref) & 1 (Ref) \\
\hline Slept under net-no & $876(30 \%)$ & $20(38 \%)$ & 1.4 (0.8 to 2.5$)$ & $457(62 \%)$ & 3.8 (3.2 to 4.5$)$ & 4.8 (3.9 to 6.0$)$ \\
\hline IRS-yes ** & 2059 (70\%) & 43 (81\%) & 1 (Ref) & $492(66 \%)$ & 1 (Ref) & 1 (Ref) \\
\hline IRS-no & $879(30 \%)$ & 10 (19\%) & 0.5 (0.3 to 1.0$)$ & $251(34 \%)$ & $1.2(1.0$ to 1.4$)$ & 1.2 (1.0 to 1.5$)$ \\
\hline No travel last month & $2658(90 \%)$ & 50 (94\%) & 1 (Ref) & 347 (47\%) & 1 (Ref) & 1 (Ref) \\
\hline $\begin{array}{l}\text { Travel inside Zanzibar } \\
\text { last month }\end{array}$ & $233(8 \%)$ & $2(4 \%)$ & 0.5 (0.1 to 1.5$)$ & $34(5 \%)$ & 1.1 (0.8 to 1.6$)$ & 1.1 (0.7 to 1.6$)$ \\
\hline $\begin{array}{l}\text { Travel outside Zanzibar } \\
\text { last month }\end{array}$ & $47(2 \%)$ & $1(2 \%)$ & 1.1 (0.1 to 5.3) & $362(49 \%)$ & 59.0 (43.1 to 82.5$)$ & $70.2(50.0$ to 100.6 \\
\hline $\begin{array}{l}\text { No travel outside Zanzibar } \\
\text { last year*** }\end{array}$ & 2811 (97\%) & 51 (98\%) & 1 (Ref) & No data & - & - \\
\hline $\begin{array}{l}\text { Travel outside Zanzibar } \\
\text { last year }\end{array}$ & $80(3 \%)$ & $1(2 \%)$ & 0.7 (0.1 to 5.1) & No data & - & - \\
\hline
\end{tabular}

*Slept last night before survey under a net

**IRS within last year before survey

***Travel from 1 month to 1 year before survey

${ }^{* * * *}$ Asymptomatic PCR negative individuals in cross-sectional survey

interventions (Additional file 2: Table S3). The mortality rate then decreased to $0.45 \%$ in 2005 after introduction of ACTs in September 2003 and was further reduced to an annual mean of $0.28 \%$ in $2007 / 2008$ after introduction of ITNs and IRS (Fig. 3). In 2009-2014, the mean rate was 0.32 . This corresponds to a $72 \%$ (95\% CI 6579) reduction between 2002 and 2007 and estimated under
5 mortalities of $51(2001 / 2)$ and $14(2007 / 8)$ per 1000 births. The observed decrease was most pronounced in children aged 1-4 years with an estimated $79 \%$ reduction from an annual mortality rate of 0.53 to $0.11 \%$ in 2001 / 2002 and 2007/2008 respectively. For children < 1 year, the corresponding reduction was $67 \%$ from 2.93 to $0.96 \%$ annual mortality rates.

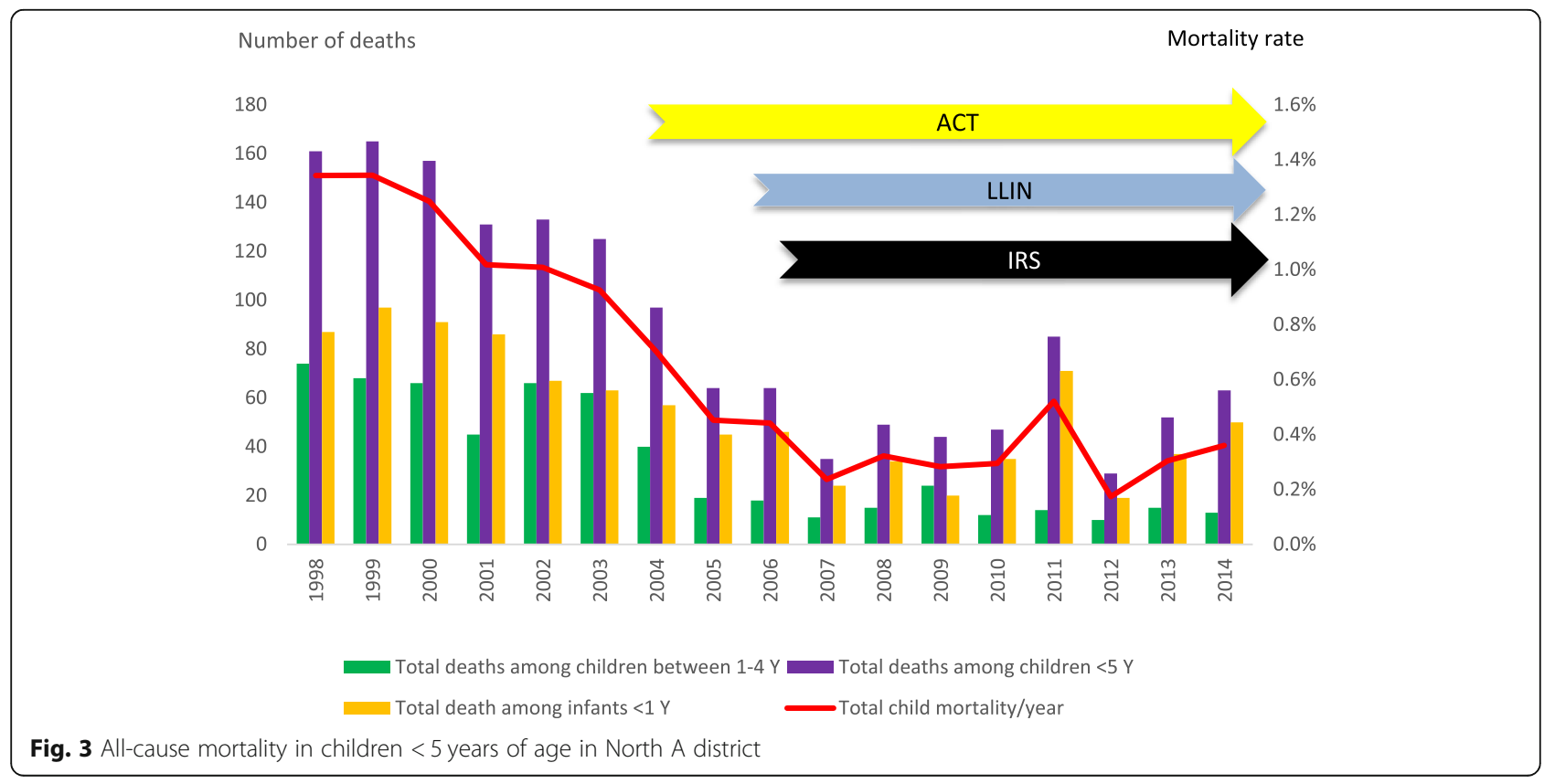




\section{Seroconversion and force of infection}

Seroprevalences to $P$. falciparum antigens increased with age in both settings (Fig. 4a, b). Profile likelihood analysis identified that models with two SCRs fitted better than a single force of infection with a change at approximately 5 years of age in the 2009 data and approximately 13 years of age in the 2015 data, consistent with the scale up of interventions between 2003 and 2006 (Fig. 4a, b). Estimates of force of infection in 2015 suggest that current SCRs are 10 and 16 fold $(90 \%, 94 \%)$ lower than before interventions: North A previous SCR 0.06 year $^{-1}$ (95\% CI 0.04-0.09), current SCR 0.006 year $^{-1}$ (0.004-0.008), and Micheweni previous SCR 0.16 year. $^{-1}(0.11-0.23)$, current SCR 0.010 year. $^{-1}(0.007-0.014)$. The average

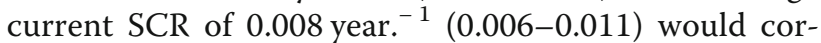
respond to an estimated annual parasite incidence (API) of about 8 cases (between 5 and 20) per 1000 inhabitants.

\section{Entomological findings}

In 2005, before the intensified vector control interventions among 2203 Anopheline mosquitoes collected in the sentinel sites of Unguja and Pemba, 2187 (99\%) were An. gambiae s.l., 11 An funestus and 5 An coustani. In 2010/2011 among 2837 collected anophelines in Micheweni and North A, 2702 (95\%) were An. gambiae s.l. and 94 (3\%) An. funestus. Other species included $A n$. rivulorum $(n=19)$, An. hancocki $(n=8)$, An. zeimani $(n=8)$ and An. maculipalpis $(n=6)$. Among An. gambiae s.l., there has been a shift in the longitudinal sentinel sites from relatively few An. arabiensis in 2005 (6/160; $4 \%$ ) to becoming predominant 2007-2014 (2907/ 3191; 91\%) supporting the increasing relative importance of outdoor biting/resting mosquitoes for malaria transmission (Additional file 1: Figure S2). An. merus has been the second most frequent subspecies, with proportion ranging from 1 to $7 \%$ in different
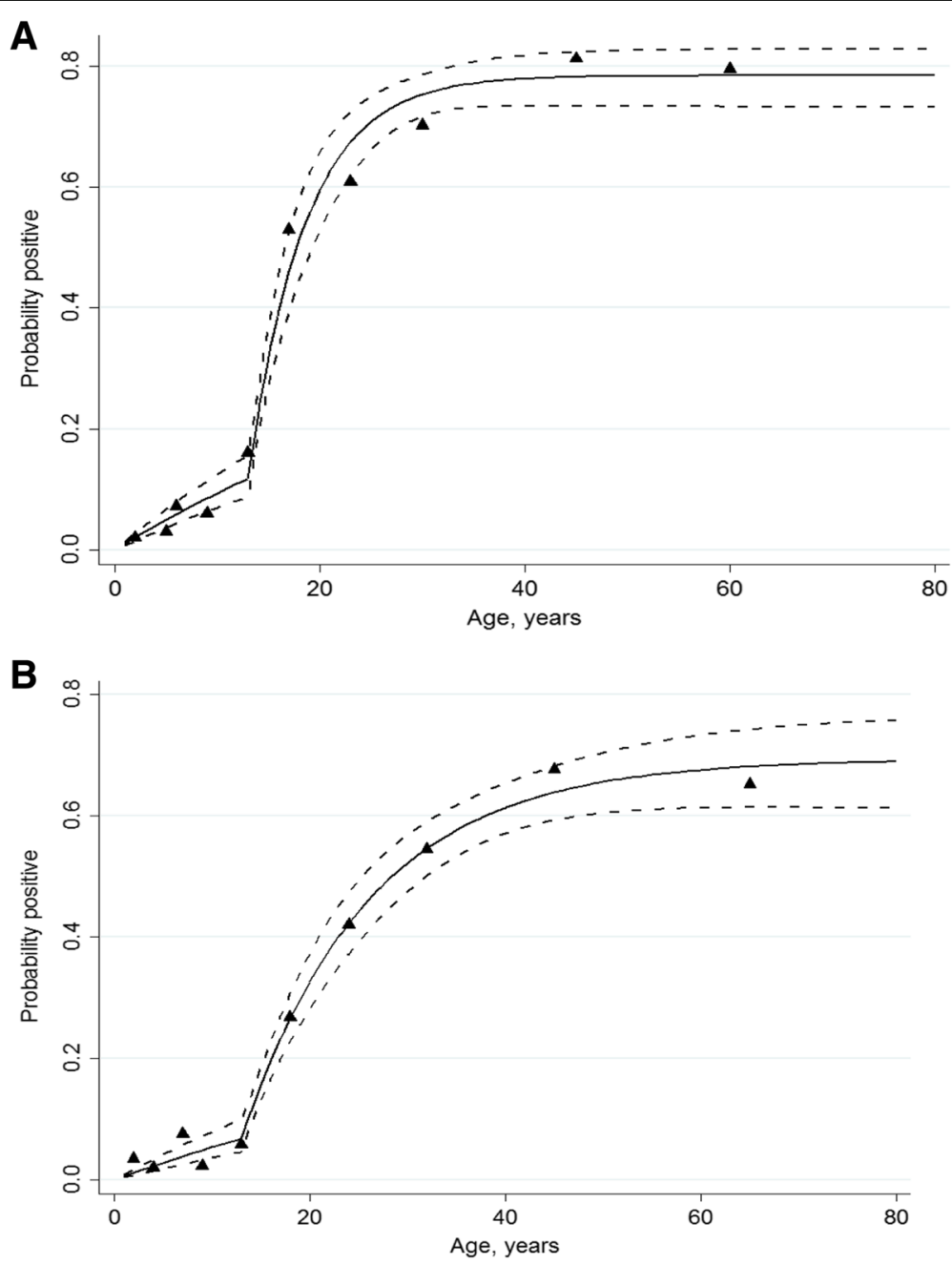

Fig. 4 Age-related prevalences of anti-P. falciparum antibodies in Micheweni district (a) and North A district (b) 
collections and years. Larvae collections in 2012/ 2013 provided similar findings with 1190/1382 (86\%) An. arabiensis and 113/1382 (8\%) An. merus.

In 2005, the mean Anopheline HBRs (in- and outdoor combined) were 16.30 and 8.57 (mean 12.44) bites/man night during January to June in Pemba and Unguja, respectively, before the first LLIN distribution. With an average sporozoite rate of 3.0\% (36/1194), the estimated annual EIRs were 178 and 94 (mean 136) infected bites respectively, with large fluctuations during the year. In 2015, the mean HBRs were 0.39 and 0.14 (mean 0.26) on the two islands (Table 4). The indoor HBRs were 0.02 and 0.13 (mean 0.08) bites/man night whereas the outdoor HBRs were 0.75 and 0.14 (mean 0.45). Assuming a correlation between sporozoite rates and asexual parasite rates and densities [22], the sporozoite rate in 2015 may be possibly estimated to $60(20 \times 3)$ times lower than in 2005 , i.e. a mean of $0.05 \%$. The annual EIRs in 2015 would then be 0.07 and 0.03 (mean 0.05) on Pemba and Unguja islands respectively.

The average reduction in overall HBR between 2005 and 2015 maybe estimated to $97.8 \%$ (Table 4). The estimated mean annual EIRs then decreased from 136 to 0.05 infected bites (Table 4), i.e. over 2000-fold (> 99.9\%). In 2015, most bites (65\%) occurred during three peak months (November and April/May) compared to 52\% during the same months in 2005.

Pyrethroid resistance was first detected in 2010 in Pemba [18] and has now spread throughout Zanzibar [19]. Hence, resistance tests performed 2012-2014 with different pyrethroids resulted in 24 to $88 \%$ and 33 to $82 \% 24 \mathrm{~h}$ (mortality of the main vectors in Unguja and Pemba, respectively ([15], and new data). In contrast, bendiocarb and pirimiphos-methyl (Actellic 300CS, Syngenta) were still $100 \%$ effective against the malaria vectors in 2014 [18].

\section{Rainfall and malaria}

The annual rainfall was rather consistent in North A between 1999 and 2015, with the exception of 2003, 2010 and 2015 (Fig. 2a-c). The mean annual rainfalls for these 3 years were $48 \%, 28 \%$ and $36 \%$ lower than the mean annual rainfall $(1345 \mathrm{~mm})$. A significant correlation was observed between monthly rainfalls and confirmed malaria diagnoses from 2007 to 2015 (Pearson correlation coefficient $[\mathrm{rp}]=0.37, p<0.01)$, but not in the previous period of monitoring (1999-2006) ([rp] $=0.04, p=0.78$ and $[\mathrm{rp}]=0.33, p=0.11$ ).

\section{Discussion}

This study provides an explicitly detailed description of the decline in malaria transmission in two districts of Zanzibar over a 12-year period. Reductions of more than 90\% were observed in prevalence of infection, incidence of malaria and human biting rate. A major drop in child mortality was seen already following the introduction of ACTs in 2003, whereas the major drop in malaria transmission occurred after the introduction of vector control in 2005.

\section{Coverage and sustainability of malaria control interventions}

An overall good access to public health care, a continuous supply and adherence to RDTs and ACTs [23-25] as well as sustained ACT efficacy $[26,27]$ have supported a continued efficient management of clinical malaria episodes in all age groups.

Quite high and sustained coverage of effective vector control has been achieved, generally higher than in other sub-Saharan countries according to their national reports [1]. Iterated household level distributions of LLINs with health information have resulted in consistently high degrees of reported use in children $<5$ years and other age groups. Combined with high coverage of IRS, this appears to have had a significant (almost 100-fold) effect on the indoor vectorial capacity, as may be forecasted [28, 29]. Both interventions are publically perceived as beneficial against malaria and biting insects in general [30, 31]. However, the recent increase in pyrethroid resistance represents a major concern [19]. The more costly carbamate or pirimiphos-methyl has therefore replaced pyrethroids in IRS whereas the pyrethroid impregnated LLINs may still provide relative protection $[32,33]$. Additionally, the change in biting behaviour of malaria vectors suggests outdoor malaria transmission poses another challenge to malaria elimination in Zanzibar. A similar shift in species proportions has been reported in other areas of Africa following wide-scale LLINs use and IRS programmes [34-37]. Complementary antivectorial efforts targeting both pyrethroid resistant and outdoor resting mosquitoes are clearly required for successful malaria elimination in Zanzibar.

\section{Malaria transmission impact}

The initial rapid reduction in transmission from 2005 to 2007 in the two study districts [8] (annual Rc about 0.5) was followed by a much slower decline with persistent low-level transmission from 2008 onwards. Although we focussed on two districts only, they appeared to be representative for all of Zanzibar. The malaria positivity rates in febrile patients reported from the health facilities in the other eight districts were quite similar to the rates in the two study districts during the study period [14]. A new equilibrium of malaria pre-elimination stage transmission thus appears established despite continuing high community uptake of the conventional control interventions. 
The reductions in malaria indices in Zanzibar are much more pronounced (around 5- to 10-fold higher) than those reported from other areas/countries of sub-Saharan Africa including Tanzania mainland [1, 3843]. In addition, these gains appear to be more sustained in Zanzibar than in many other areas where a tendency of resurgence may be occurring in the latest years [1]. This highlights the uniqueness of the malaria elimination efforts in Zanzibar. There are probably multiple reasons for the effectiveness but we believe a major factor is higher population-level uptake of the interventions due in large part to the strong commitment of ZAMEP and the Zanzibar Government and strong involvement of the communities. An easy access to health care and accurate malaria treatment is probably also essential. We do not believe other general factors such as socioeconomic changes and sudden improvement in health care may have strongly influenced the impact on the rapid 10 - to 100-fold reduction in malaria indices. A socio-economic development has probably occurred in Zanzibar as in many other areas of Africa during the study period, but would only account for a minor part of the malaria control impact.

The establishment of this new persistent level of low transmission contrasts with the malaria elimination feasibility report for Zanzibar based on mathematical modelling which predicted a possible annually continuous Rc of 0.5 and elimination achieved by 2020 if effective intervention coverage was kept at approximately $75 \%$ [44]. There may be several reasons why this prediction became unrealistic. Firstly, malaria transmission is now more seasonal and geographically heterogeneous with clear foci of infection [14], which may be driving current transmission [45]. Secondly, sensitive molecular testing by PCR has highlighted the major reservoir of low-density asymptomatic parasitaemias across all age groups, which appears consistent with other low transmission areas [46-48]. Importantly, these low-density parasitaemias may contribute significantly to the residual ongoing transmission $[49,50]$ and interestingly this appears to be possible despite a major decline in HBRs (Table 4). An additional interesting finding was that the relative proportion of low-density $P$. malariae infections increased after the initiation of interventions up to 2011. This may reflect greater longevity of untreated asymptomatic P. malariae than P. falciparum infections [51] but relatively lower presently ongoing transmission.

Thirdly, imported malaria may represent a major hindrance for elimination. A relative risk factor for clinical malaria infection was indeed history of travel outside Zanzibar. Such travel, mainly to/from Tanzania mainland, was reported by $49 \%$ of clinical malaria patients (OR 70) in 2015. Neither recent nor previous travel were significant risk factors for asymptomatic infections. In
2010, travel outside Zanzibar was reported by only 9/ 121 (7\%) malaria-confirmed patients (OR 9) [23]. In 2013, travel outside of Zanzibar was reported by $30 \%$ of reported clinical malaria patients [14]. A tentative interpretation of this may be that an increasingly important fraction of new clinical infections are acquired from outside Zanzibar although still somewhat less than reported estimates for Zanzibar based on modelling [52]. Conversely our cross-sectional data, including the serology results, may suggest that many infections are locally acquired and possibly asymptomatic and thus remain as untreated residual infections for a significant period of time allowing for maintained residual local transmission [53]. Hence, the official figures in Zanzibar of approximately 3000 malaria confirmed clinical infections significantly underestimate the actual incidence of newly acquired infections. Such figures may be better reflected by SCR estimates. Extrapolating the mean SCR for the two study districts $\left(0.008\right.$ year $\left.^{-1}\right)$ to whole of Zanzibar would suggest over 10,000 new infections annually.

A fourth reason for the halt in malaria transmission reduction despite the significant reduction of HBRs is probably that the residual vector population mainly biting and resting outdoors is now less affected by indoor vector control. Hence, not having IRS recently performed was not identified as a major risk factor, while not sleeping under LLIN was only a risk factor for clinical malaria, again supporting that most transmission may occur outdoors. It also suggests that the preventive mass effects of LLIN use and IRS on indoor transmission are now more significant than the individually preventive effects. However, the spreading resistance to pyrethroids [19] and more restricted coverage of IRS represent challenges for future sustained impact, although the exact epidemiological effects of resistance to the insecticide in the nets appear to vary $[32,33]$, and indoor HBRs presently remain very low in our study area.

A fifth potential challenge is that malaria immunity is expected to decline as transmission reduces [54]. An increased proportion of clinical malaria episodes was seen among patients $>5$ years although such age shift was not yet seen among PCR detected low-density parasitaemias. These older age groups were previously likely to be clinically immuno-protected through previous repeated exposure. A reason for the relative age shift may however also be behavioural. Older age groups who remain outside in the evening are exposed to more outdoor biting mosquitoes and may be less prone to use LLINs against mosquitoes biting indoor. Finally, it may also result from more frequent travel to mainland Tanzania by older age groups.

It is commonly stated that it is easier to control/eliminate malaria on an island (ex Zanzibar) than in-country 
(e.g. Tanzania mainland). This is may be true for a small island with a small population [55]. However, we do not believe this to be a major reason for the high impact on the two large islands of Zanzibar with populations over half a million each. There is obviously increased risk of imported malaria on the African continent between neighbouring countries through more crossing of borders as well as to some extent exchange of mosquitoes to nearby areas. But grossly besides the very border areas there should not be major differences between the malaria control efforts required in Zanzibar islands and mainland Tanzania. We therefore consider the findings and challenges in Zanzibar highly applicable to other African countries.

\section{Public health impact}

The study data provide evidence of a major improvement in child health. A highly significant reduction of all cause child mortality coincided with the introduction of ACT whereas the most significant reduction in malaria incidence occurred after the intensified vector control. Although there was a trend of reduction in under 5 child mortality already before 2003, the decline 2003-2005 was clearly more pronounced.

The reported child deaths to the Vital Registry probably represent an underestimated mortality but we believe the reporting rate remained rather similar during years of study, allowing for reasonably valid trend analysis. In addition, the specifically large mortality reduction during 2003-2006 cannot be explained by any other public health intervention at that time in Zanzibar. A major decrease in crude child mortality was also observed on Bioko Island after massive malaria control interventions [42]. In parallel to our observed mortality reduction, there was also reduced hospitalisation for severe malaria [56] and major reduction in severe anaemia requiring blood transfusions to $<5$ children [8], a common severe manifestation of malaria in sub-Saharan Africa. The overall reduction of clinical malaria episodes resulted in a decline in fever episodes especially in children, also noted by their caretakers and thus a good incentive for sustained LLIN use [30, 31].

The strong impact on crude mortality may largely be explained directly by reduced malaria specific mortality from ACT preventing development of severe malaria manifestations and the vector control reducing malaria incidence. It may however also result indirectly from the general reduction of malaria infection and its associated anaemia, being a risk factor for severe manifestations of other concomitant bacterial infections [57], e.g. septicaemia [58] or pneumonia.

\section{Additional tools and strategies required}

A new low malaria transmission epidemiology has emerged in Zanzibar with spatial, temporal and demographic foci of infection. These foci are likely to be influenced by outdoor transmission and increasing insecticidal resistance, a substantial asymptomatic parasite reservoir and an apparent increasing number of imported infections. All this necessitates the addition of new malaria control tools and strategies.

Screening (by RDT) and subsequent preventive treatment of asymptomatic but possible parasite carriers has now been introduced in households where clinical malaria episodes have been identified and potentially in the future within for example 300-m radius [59]. Since 2012, IRS with carbamate and pirimiphos-methyl has been specifically targeting identified hotspots and larvicing interventions are being trialled in selected foci. Gametocytocidal single low-dose primaquine is being introduced along with ACT. General surveillance is also being reinforced by more comprehensive and regular epidemiological investigations of newly detected and reported cases, as well as more comprehensive monitoring of entomological insecticide resistance and parasitological drug resistance.

However, other possibly more aggressive approaches are also needed. This may include screen and treat strategies potentially including new highly sensitive diagnostics [60-62] or targeted mass/focal drug administration $[59,63]$ possibly including seasonal chemoprevention $[64,65]$. These actions may be targeted to hotspot areas [66] and/or population groups at risk of residual parasite reservoir. Additional vector control targeting $A n$. arabiensis populations needs to be implemented, e.g. different outdoor mosquito "attract and kill" methods. Case detection and response also needs further development to ensure future rapid prevention of outbreaks especially from imported infections. Since imported malaria represents a significant barrier to malaria control and elimination efforts in Zanzibar, several additional preventive strategies may be considered, especially during high transmission season. Such interventions may include chemoprophylaxis to Zanzibar is travelling to mainland, and mass screening or presumptive treatment of anyone arriving from mainland. However, formal and informal exit and/or entry points from/to Zanzibar are numerous and the efficiency of for example screening and treatment may not be very significant with standard diagnostic tools [61]. This will be explored in Zanzibar in the near future.

With old reinforced and new introduced interventions, the Rc presently around 1 may possibly be reduced to 0.5 again. The presently annually reported 3000 clinical malaria cases and our estimated 10,000 infections would then be reduced below 10 and a state of elimination potentially achieved by approximately 2026 .

\section{Conclusions}

Zanzibar has made huge progress towards malaria elimination, differently from most other areas in sub-Saharan 
Africa where recent progress appears difficult to sustain. However, bending the transmission curve further requires significant thought, impetus and funding. In the past, there have been attempts to strongly control/eliminate malaria in Zanzibar although not as successfully as now, but malaria has resurged each time due to high vulnerability and receptivity. Zanzibar is therefore now embarking on a new roadmap. The data presented and the obstacles and challenges identified in the present study suggest that responding to these challenges with reoriented and new strategies Zanzibar may possibly accelerate the reduction and elimination of local malaria transmission within a coming 10 -year period. This may then provide a highly wishful proof of elimination concept from a high endemic area. However, clearly, the global community presently expresses overoptimistic ambitions regarding the timing of malaria elimination, relying on views and modelling which grossly underestimate the challenges ahead related to both escaping mechanisms of the parasite and mosquito as well as operational constraints.

\section{Additional files}

Additional file 1: Figure S1. Proportions of $<5$ and $\geq 5$ years of age reporting having slept under a bed net the night before and proportion of households reporting having been sprayed the year before the respective surveys between 2003 to 2015 in a) Micheweni district and b) North A district. Figure S2 Proportions of An. arabiensis among An gambiae s.I mosquito samples collected in surveys on Unguja island, Zanzibar between 2005 and 2014. (DOCX 100 kb)

Additional file 2: Table S1. Community prevalences of $P$. falciparum asexual parasitaemia and gametocytaemia according to microscopy of RDT by age group in North A and Micheweni districts of Zanzibar, MayJune 2003 to 2015. Table S2 Proportions of confirmed malaria patients (all age groups) among tested patients attending public health care facilities in Micheweni and North A districts between 1999 and 2015. Table S3 All-cause mortality in children < 5 years of age in North A district between 1998 and 2014. Data from Vital Registry. (DOCX 20 kb)

\section{Abbreviations}

ACT: Artemisinin-based combination therapy; AL: Artemether-lumefantrine; AMFm: Affordable medicines for malaria; API: Annual parasite incidence (per 1000 inhabitants); ASAQ: Artesunate-amodiaquine; HBR: Human biting rate; HRP2: Histidine-rich-protein 2; IRS: Indoor residual spraying; ITN: Insecticidetreated net; LLIN: Long-lasting insecticidal net; MCN: Malaria case notification (system); OD: Optical density; RDT: Rapid diagnostic test; SCR: Seroconversion rate; WHO: World Health Organization; ZAMEP: Zanzibar Malaria Elimination Programme

\section{Acknowledgements}

Not applicable.

\section{Funding}

This work received support from Family Erling Persson Foundation (AB), Swedish Research Council (AB), Swedish Civil Contingencies Agency (AM) and Wellcome Trust (CD). The funders had no role in study design, data collection, data analyses, data interpretation or writing of the report.

\section{Availability of data and materials}

The datasets used and/or analysed during the current study are available from the corresponding author on reasonable request.

\section{Authors' contributions}

$A B j, D S$ and $A M$ conceived the study and its design. ASA and MM were responsible for the interventions and assisted in the study design. DS, UM, $\mathrm{MM}, \mathrm{AKA}, \mathrm{A}-\mathrm{WA}, \mathrm{ABh}, \mathrm{RO}, \mathrm{JM}, \mathrm{KAH}, \mathrm{JC}, \mathrm{KE}$ and $\mathrm{HM}$ performed data collections. UM, BA-S, WX and CD performed and/or interpreted laboratory analyses and data. $A B j, D S, C D, U M, H M$ and $A M$ compiled data and/or interpreted the results. MCS and MP assisted in statistical analyses. ABj and DS drafted the article. All authors contributed to critical revision of the paper. All authors read and approved the final manuscript.

\section{Ethics approval and consent to participate}

Ethical approvals for different sub-studies were obtained from the Zanzibar Medical Research Ethical Committee and Regional Ethics Review Board, Stockholm, Sweden.

\section{Consent for publication}

Not applicable.

\section{Competing interests}

The authors declare that they have no competing interests.

\section{Publisher's Note}

Springer Nature remains neutral with regard to jurisdictional claims in published maps and institutional affiliations.

\section{Author details}

${ }^{1}$ Department of Microbiology, Tumor and Cell Biology, Karolinska Institutet, Solnavägen 9, SE-171 77 Stockholm, Sweden. ${ }^{2}$ Health Metrics at Sahlgrenska Academy, University of Gothenburg, Gothenburg, Sweden. ${ }^{3}$ Zanzibar Malaria Elimination Programme, Zanzibar, Tanzania. ${ }^{4}$ MEASURE Evaluation, Dar es Salaam, Tanzania. ${ }^{5}$ London School of Hygiene and Tropical Medicine, London, UK. ${ }^{6}$ Department of Infectious Diseases, University of Gothenburg, Gothenburg, Sweden. ${ }^{7}$ Centre for Applied Biostatistics, University of Gothenburg, Gothenburg, Sweden. ${ }^{8}$ Biostatistics Unit, Institute of Environmental Medicine, Karolinska Institutet, Stockholm, Sweden. ${ }^{9}$ Training and Research, Mnazi Mmoja Hospital, Zanzibar, Tanzania. ${ }^{10}$ Department of Women's and Children's Health, International Maternal and Child Health, Uppsala University, Uppsala, Sweden.

Received: 29 June 2018 Accepted: 17 December 2018

Published online: 22 January 2019

\section{References}

1. WHO. World Malaria Report 2017. Available at: http://www.who.int/malaria/ publications/world_malaria_report_2018. Accessed 19 Nov 2018.

2. Maintaining momentum for malaria elimination. Lancet 2009; 374(9686): 266.

3. Feachem RG, Phillips AA, Hwang J, et al. Shrinking the malaria map: progress and prospects. Lancet. 2010;376(9752):1566-78.

4. Mendis K, Rietveld A, Warsame M, Bosman A, Greenwood B, Wernsdorfer WH. From malaria control to eradication: the WHO perspective. Trop Med Int Health. 2009;14(7):802-9.

5. World Health Organization, Global Malaria Programme 2007. Malaria elimination: a field manual for low and moderate endemic countries. WHO Report.

6. Metselaar D, Thiel v. Classification of malaria. Trop Geogr Med. 1959;11:157-61.

7. Schwartz E, Pener H, Issa SM, Golenser J. An overview of the malaria situation in Zanzibar. J Community Health. 1997;22(1):33-44.

8. Bhattarai A, Ali AS, Kachur SP, et al. Impact of artemisinin-based combination therapy and insecticide-treated nets on malaria burden in Zanzibar. PLoS Med. 2007;4(11):e309.

9. Smith DL, Hay SI. Endemicity response timelines for Plasmodium falciparum elimination. Mal J. 2009;8:87.

10. Wooden J, Kyes S, Sibley CH. PCR and strain identification in Plasmodium falciparum. Parasitol Today. 1993;9(8):303-5.

11. Xu W, Morris U, Aydin-Schmidt B, et al. SYBR green real-time PCR-RFLP assay targeting the Plasmodium cytochrome $B$ gene - a highly sensitive molecular tool for malaria parasite detection and species determination. PLoS One. 2015;10(3):e0120210.

12. Kamau $E$, Tolbert $L S$, Kortepeter $L$, et al. Development of a highly sensitive genus-specific quantitative reverse transcriptase real-time PCR assay for 
detection and quantitation of plasmodium by amplifying RNA and DNA of the 18S rRNA genes. J Clin Microbiol. 2011;49(8):2946-53.

13. Stewart L, Gosling R, Griffin J, et al. Rapid assessment of malaria transmission using age-specific sero-conversion rates. PLoS One. 2009;4(6):e6083.

14. Zanzibar Malaria Control (Elimination) Programme. Zanzibar Malaria Epidemic Early Detection System and Entomological Surveillance. Ministry of Health and Social Welfare. Annual and biannual reports 2005-2016.

15. Jones $\mathrm{CM}$, Haji KA, Khatib BO, et al. The dynamics of pyrethroid resistance in Anopheles arabiensis from Zanzibar and an assessment of the underlying genetic basis. Parasit Vectors. 2013;6:343.

16. Beier JC, Perkins PV, Koros JK, et al. Malaria sporozoite detection by dissection and ELISA to assess infectivity of afrotropical Anopheles (Dipteria: Culicidae). J Med Entomol. 1990;27:377-84.

17. WHO. Test procedures for insecticide resistance monitoring in malaria vector mosquitoes. Geneva: World Health Organization; 2016. SBN 9789241511575

18. Haji KA, Thawer NG, Khatib BO, et al. Efficacy, persistence and vector susceptibility to pirimiphos-methyl (Actellic 300CS) insecticide for indoor residual spraying in Zanzibar. Parasit Vectors. 2015;8:628.

19. Haji KA, Khatib BO, Smith $\mathrm{S}$, et al. Challenges for malaria elimination in Zanzibar: pyrethroid resistance in malaria vectors and poor performance of long-lasting insecticide nets. Parasit Vectors. 2013;6:82.

20. Drakeley CJ, Corran PH, Coleman PG, et al. Estimating medium- and longterm trends in malaria transmission by using serological markers of malaria exposure. Proc Natl Acad Sci U S A. 2005;102(14):5108-13.

21. Cook J, Kleinschmidt I, Schwabe C, et al. Serological markers suggest heterogeneity of effectiveness of malaria control interventions on Bioko Island, Equatorial Guinea. PLoS One. 2011;6(9):e25137.

22. Ross A, Killeen G, Smith T. Relationships between host infectivity to mosquitoes and asexual parasite density in Plasmodium falciparum. Am J Trop Med Hyg. 2006;75(suppl2):32-7.

23. Shakely $D$, Elfving $K$, Aydin-Schmidt $B$, et al. The usefulness of rapid diagnostic tests in the new context of low malaria transmission in Zanzibar. PLoS One. 2013:8(9):e72912.

24. Msellem Ml, Martensson $\mathrm{A}$, Rotllant $\mathrm{G}$, et al. Influence of rapid malaria diagnostic tests on treatment and health outcome in fever patients, Zanzibar: a crossover validation study. PLoS Med. 2009;6(4):e1000070.

25. Beer N, Ali AS, Rotlant $\mathrm{G}$, et al. Adherence to artesunate-amodiaquine combination therapy for uncomplicated malaria in children in Zanzibar, Tanzania. Trop Med Int Health. 2009;7:766-74.

26. Martensson A, Stromberg J, Sisowath C, et al. Efficacy of artesunate plus amodiaquine versus that of artemether-lumefantrine for the treatment of uncomplicated childhood Plasmodium falciparum malaria in Zanzibar, Tanzania. Clin Infect Dis. 2005;41(8):1079-86.

27. Froberg $G$, Jornhagen $L$, Morris $U$, et al. Decreased prevalence of Plasmodium falciparum resistance markers to amodiaquine despite its wide scale use as ACT partner drug in Zanzibar. Malar J. 2012:11:321.

28. Howard SC, Omumbo J, Nevill C, Some ES, Donnelly CA, Snow RW. Evidence for a mass community effect of insecticide-treated bednets on the incidence of malaria on the Kenyan coast. Trans R Soc Trop Med Hyg. 2000; 94(4):357-60.

29. Killeen GF, Smith TA, Ferguson HM, et al. Preventing childhood malaria in Africa by protecting adults from mosquitoes with insecticide-treated nets. PLoS Med. 2007;4(7):e229.

30. Beer N, Ali AS, de Savigny D, et al. System effectiveness of a targeted free mass distribution of long lasting insecticidal nets in Zanzibar, Tanzania. Malaria J. 2010:9:173.

31. Beer N, Ali AS, Shakely D, et al. High effective coverage of vector control interventions in children after achieving low malaria transmission in Zanzibar, Tanzania. Malaria J. 2013;12:38.

32. Ranson H, N'Guessan R, Lines J, Moiroux N, Nkuni Z, Corbel V. Pyrethroid resistance in African anopheline mosquitoes: what are the implications for malaria control? Trends Parasitol. 2011;27(2):91-8.

33. Wondji CS, Coleman M, Kleinschmidt I, et al. Impact of pyrethroid resistance on operational malaria control in Malawi. Proc Natl Acad Sci U S A. 2012; 109(47):19063-70.

34. Bayoh MN, Mathias DK, Odiere MR, et al. Anopheles gambiae: historical population decline associated with regional distribution of insecticidetreated bed nets in western Nyanza Province, Kenya. Malaria J. 2010;9:62.

35. Derua YA, Alifrangis M, Hosea KM, et al. Change in composition of the Anopheles gambiae complex and its possible implications for the transmission of malaria and lymphatic filariasis in North-Eastern Tanzania. Malar J. 2012:11:188.

36. Moiroux N, Gomez MB, Pennetier C, et al. Changes in Anopheles funestus biting behavior following universal coverage of long-lasting insecticidal nets in Benin. J Infect Dis. 2012;206(10):1622-9.

37. Mwangangi $\mathrm{JM}$, Mbogo $\mathrm{CM}$, Orindi $\mathrm{BO}$, et al. Shifts in malaria vector species composition and transmission dynamics along the Kenyan coast over the past 20 years. Malar J. 2013;12:13.

38. Noor AM, Kinyoki DK, Mundia CW, et al. The changing risk of Plasmodium falciparum malaria infection in Africa: 2000-10: a spatial and temporal analysis of transmission intensity. Lancet. 2014;383(9930):1739-47.

39. Ceesay SJ, Casals-Pascual C, Nwakanma DC, et al. Continued decline of malaria in the Gambia with implications for elimination. PLoS One. 2010; 5(8):e12242.

40. Karema C, Aregawi MW, Rukundo A, et al. Trends in malaria cases, hospital admissions and deaths following scale-up of anti-malarial interventions, 2000-2010, Rwanda. Malaria J. 2012:11:236.

41. Mmbando BP, Vestergaard LS, Kitua AY, Lemnge MM, Theander TG, Lusingu JP. A progressive declining in the burden of malaria in North-Eastern Tanzania. Malar J. 2010;9:216.

42. Kleinschmidt I, Schwabe C, Benavente L, et al. Marked increase in child survival after four years of intensive malaria control. Am J Trop Med Hyg. 2009;80(6):882-8.

43. Trape JF, Tall A, Sokhna C, et al. The rise and fall of malaria in a west African rural community, Dielmo, Senegal, from 1990 to 2012: a 22 year longitudinal study. Lancet Infect Dis. 2014;14(6):476-88.

44. Zanzibar Malaria Control Programme. Malaria elimination in Zanzibar a feasibility assessment. Zanzibar: Ministry of Health and Social Welfare; 2009.

45. Ahmed S, Galagan S, Scobie H, et al. Malaria hotspots drive hypoendemic transmission in the Chittagong Hill Districts of Bangladesh. PLoS One. 2013; 8(8):e69713.

46. Harris I, Sharrock WW, Bain LM, et al. A large proportion of asymptomatic Plasmodium infections with low and sub-microscopic parasite densities in the low transmission setting of Temotu Province, Solomon Islands: challenges for malaria diagnostics in an elimination setting. Malaria J. 2010;9:254.

47. Manjurano A, Okell L, Lukindo T, et al. Association of sub-microscopic malaria parasite carriage with transmission intensity in North-Eastern Tanzania. Malar J. 2011;10:370.

48. Okell LC, Bousema T, Griffin JT, Ouedraogo AL, Ghani AC, Drakeley CJ. Factors determining the occurrence of submicroscopic malaria infections and their relevance for control. Nat Commun. 2012;3:1237.

49. Ouedraogo AL, Bousema T, Schneider P, et al. Substantial contribution of submicroscopical Plasmodium falciparum gametocyte carriage to the infectious reservoir in an area of seasonal transmission. PLoS One. 2009; 4(12):e8410.

50. Schneider P, Bousema JT, Gouagna LC, et al. Submicroscopic Plasmodium falciparum gametocyte densities frequently result in mosquito infection. Am J Trop Med Hyg. 2007;76(3):470-4.

51. Bretscher MT, Maire N, Felger I, Owusu-Agyei S, Smith T. Asymptomatic Plasmodium falciparum infections may not be shortened by acquired immunity. Malar J. 2015:14:294.

52. Le Menach A, Tatem AJ, Cohen JM, et al. Travel risk, malaria importation and malaria transmission in Zanzibar. Sci Rep. 2011:1:93.

53. Björkman A. Asymptomatic low-density malaria infections: a parasite strategy survival? Lancet Infect Dis. https://doi.org/10.1016/S14733099(18)30047-1.

54. O'Meara WP, Mwangi TW, Williams TN, McKenzie FE, Snow RW, Marsh K. Relationship between exposure, clinical malaria, and age in an area of changing transmission intensity. Am J Trop Med Hyg. 2008;79(2):185-91.

55. Kaneko A, Taleo G, Kalkoa M, Yamar S, Kobayakawa T, Bjorkman A. Malaria eradication on islands. Lancet. 2000;356(9241):1560-4.

56. Aregawi MW, Ali AS, Al-mafazy AW, et al. Reductions in malaria and anaemia case and death burden at hospitals following scale-up of malaria control in Zanzibar, 1999-2008. Malar J. 2011:10:46.

57. Molineaux L. Nature's experiment: what implications for malaria prevention? Lancet. 1997:349(9066):1636-7.

58. Scott JA, Berkley JA, Mwangi I, et al. Relation between falciparum malaria and bacteraemia in Kenyan children: a population-based, case-control study and a longitudinal study. Lancet. 2011;378(9799):1316-23.

59. Björkman A, Cook J, Sturrock H, et al. Spatial distribution of falciparum malaria infections in Zanzibar: implications for focal drug administration 
strategies targeting asymptomatic parasite carriers. Clin Infect Dis. 2017;64: 1236-43.

60. Cook J, Xu W, Msellem M, Vonk M, et al. Mass screening and treatment on the basis of results of a Plasmodium falciparum-specific rapid diagnostic test did not reduce malaria incidence in Zanzibar. J Infect Dis. 2015;211:1476-83.

61. Aydin-Schmidt B, Morris U, Ding XC, et al. Field evaluation of a high throughput loop mediated isothermal amplification test for the detection of asymptomatic plasmodium infections in Zanzibar. PLoS One. 2017;12(1):e 0169037.

62. Morris U, Khamis M, Aydin-Schmidt B, Abass AK, Msellem MI, Nassor MH, Gonzalez IJ, Martensson A, Ali AS, Bjorkman A, et al. Field deployment of loop-mediated isothermal amplification for centralized mass-screening of asymptomatic malaria in Zanzibar: a pre-elimination setting. Malar J. 2015; 14:205.

63. Morris U, Msellem M, Mkali H, Islam A, Aydin-Schmidt B, Jovel I, Shija S, Khamis M, Mohammed Ali S, Hodzic L, Magnusson E, Poirot E, Bennett A, Sachs M, Tärning J, Mårtensson A, Ali AS, Björkman A. A cluster randomised controlled trial of two rounds of mass drug administration in Zanzibar, a malaria pre-elimination setting - high coverage and safety, but no significant impact on transmission. Submitted to BMC medicine on $9^{\text {th }}$ July 2018.

64. Greenwood B. Review: intermittent preventive treatment--a new approach to the prevention of malaria in children in areas with seasonal malaria transmission. Trop Med Int Health. 2006;11(7):983-91.

65. WHO Global Malaria Programme. Seasonal malaria chemoprevention (SMC). Available at: http://www.who.int/malaria/areas/preventive_therapies/ children/en/. Accessed 1 May 2017.

66. Bousema T, Griffin JT, Sauerwein RW, et al. Hitting hotspots: spatial targeting of malaria for control and elimination. PLoS Med. 2012;9(1):e1001165.

Ready to submit your research? Choose BMC and benefit from:

- fast, convenient online submission

- thorough peer review by experienced researchers in your field

- rapid publication on acceptance

- support for research data, including large and complex data types

- gold Open Access which fosters wider collaboration and increased citations

- maximum visibility for your research: over $100 \mathrm{M}$ website views per year

At $\mathrm{BMC}$, research is always in progress.

Learn more biomedcentral.com/submissions 